\title{
sciendo
}

\section{EFFECT OF ENZYMATIC PRO-OXIDANT AND ANTIOXIDANT SYSTEMS ON BOVINE OOCYTE IN VITRO MATURATION*}

\author{
Sergio Morado ${ }^{1,2}$, Stephania Madrid Gaviria ${ }^{2}$, Gabriel Dalvit ${ }^{1}$, Pablo Cetica ${ }^{1,2}$ \\ ${ }^{1}$ Facultad de Ciencias Veterinarias, Instituto de Investigación y Tecnología en Reproducción Animal (INITRA, UBA), Universidad de Buenos \\ Aires, C1427CWO, Av. Chorroarín 280, Buenos Aires, Argentina \\ ${ }^{2}$ Instituto de Investigaciones en Producción Animal (INPA, UBA-CONICET), Universidad de Buenos Aires, C1427CWO, Av. Chorroarín 280, \\ Buenos Aires, Argentina \\ •Corresponding author: smorado@fvet.uba.ar
}

\begin{abstract}
The role of reactive oxygen species (ROS) during oocyte in vitro maturation (IVM) is still controversial. Although an increase in ROS production may cause deleterious effects in cells, these reactive species may also act as signaling molecules influencing different cell functions. The aim of this study was to examine the effect of varying endogenous ROS levels during IVM on the process of bovine oocyte maturation. To do so, different enzymatic antioxidant (catalase, or superoxide dismutase + catalase, or diphenyl iodonium) or pro-oxidant systems (xanthine + xanthine oxidase, or xanthine + xanthine oxidase + catalase) were added to the culture medium. ROS levels were determined by 2',7'-dichlorodihydrofluorescein diacetate stain, nuclear maturation was evaluated by the presence of the metaphase II chromosome configuration at $22 \mathrm{~h}$ of IVM and cleavage rate was recorded $48 \mathrm{~h}$ post-in vitro fertilization. ROS levels were only significantly increased $(\mathrm{P}<0.05)$ by the $\mathrm{O}_{2}^{--}$generating system (xanthine + xanthine oxidase + catalase), but meiotic maturation rates were significantly lower $(\mathrm{P}<0.05)$ in all the evaluated systems compared with the control, except for the diphenyl iodonium group. However, this last group presented a significantly lower $(\mathbf{P}<\mathbf{0 . 0 5})$ cleavage rate in comparison to the control group. These results indicate that $\mathrm{ROS}$ would play an essential role during oocyte maturation, since its increase or decrease beyond a physiological level significantly reduced nuclear or cytoplasmic maturation rates in bovine oocytes.
\end{abstract}

Key words: bovine oocytes, in vitro maturation, reactive oxygen species

The role of reactive oxygen species (ROS) in gametes and embryo in vitro culture and manipulation is still controversial. It has been proposed that an adequate level of hydrogen peroxide $\left(\mathrm{H}_{2} \mathrm{O}_{2}\right)$ would be necessary to increase bovine oocyte developmental competence (Pandey and Chaube, 2014; Vandaele et al., 2010) and a gradual increase in ROS levels has been observed during the first stages of embryo development (Ali et al., 2018; Lopes et al., 2010; Morado et al., 2013; Velez-Pardo et al., 2007), which could be related to metabolic changes.

On the other hand, certain studies have demonstrated the expression of genes which code for antioxidant enzymes throughout the in vitro culture of cumulus-oocyte complexes (COCs). These studies propose that the storage of antioxidants in the oocyte during its growth and maturation would be important for the developmental competence of the future embryo (Christou-Kent et al., 2020; García-Martínez et al., 2020; Mouatassim et al., 1999).
Reduction-oxidation (redox) reactions are essential for living systems displaying a dynamic equilibrium, where biologically important oxidations occur in a controlled manner (Aitken, 2020). The oxidative metabolism, essential for energy production, is directly associated with ROS generation (Carbone et al., 2003), being so that mitochondria, specifically the electron transport chain, are the major sources of superoxide anion $\left(\mathrm{O}_{2}^{-}\right)$and $\mathrm{H}_{2} \mathrm{O}_{2}$ generation (Goud et al., 2008). ROS can also be physiologically produced $\mathrm{O}_{2}{ }^{-}$as the primary function of the NADPH oxidase (NOXs) family. These enzymes transfer an electron across the cell membrane from NADPH in the cytosol to $\mathrm{O}_{2}$ in the extracellular space generating $\mathrm{O}_{2}^{-}$which can be rapidly converted to $\mathrm{H}_{2} \mathrm{O}_{2}$ ( $\mathrm{Li}$ et al., 2018). In this way, the main product of NOX is $\mathrm{H}_{2} \mathrm{O}_{2}$ which is more stable and permeable to the cell membrane (Buck et al., 2019). 
In order to maintain the balance between ROS production and removal, living organisms possess enzymatic and non-enzymatic antioxidant systems, the former being more efficient (Kala et al., 2017). Enzymatic systems include superoxide dismutase (SOD), which removes $\mathrm{O}_{2}{ }^{--}$in a dismutation reaction producing $\mathrm{H}_{2} \mathrm{O}_{2}$ and $\mathrm{O}_{2}$ (Carbone et al., 2003), and catalase and glutathione peroxidase (GSH-Px) which catalyze the removal of $\mathrm{H}_{2} \mathrm{O}_{2}$. Catalase metabolizes $\mathrm{H}_{2} \mathrm{O}_{2}$ into the non-reactive molecules $\mathrm{H}_{2} \mathrm{O}$ and $\mathrm{O}_{2}$ (Von Mengden et al., 2020), while in the GSH-Px reaction, reduced glutathione $(\mathrm{GSH})$ is oxidized to GSSG (oxidized glutathione), which in turn can be reduced by the action of glutathione reductase (GSSG-Rx) using NADPH (Carbone et al., 2003).

ROS have been proposed to act in several signaling pathways, since they are small, they can diffuse short distances and there are several rapid and controllable mechanisms for their production and removal. This way, ROS, especially $\mathrm{H}_{2} \mathrm{O}_{2}$, may influence cell proliferation, cell death and gene expression (Altenhöfer et al., 2015; Hancock et al., 2001). Nevertheless, an excess in ROS production may cause deleterious effects in cells. VelezPardo et al. (2007) reported that the arrest in embryo development is associated with an increased production of $\mathrm{O}_{2}^{-}$and $\mathrm{H}_{2} \mathrm{O}_{2}$ which causes alterations in the mitochondrial membrane potential, with subsequent activation of the proteases/caspases pathway resulting in apoptosis. In mouse oocytes, the exposure to $\mathrm{O}_{2}^{--}$increased zona pellucida dissolution time, altered ooplasm microtubule dynamics and increased cortical granule loss (Goud et al., 2008), while in porcine oocytes an increased ooplasmic ROS content is related with a higher mRNA abundance of acetyltransferase gene HAT1 and increased levels of acetylation of histone $\mathrm{H} 4$ at lysine 12 , associated with oocyte aging (Cui et al., 2011). In the follicular fluid of developing oocytes, enzymatic antioxidants work in concert with non-enzymatic antioxidants, such as GSH, vitamin $\mathrm{C}$, vitamin $\mathrm{E}$ and taurine, presumably to counteract the potentially harmful effects of ROS (El-Shahat and Kandil, 2012; Gupta et al., 2011; Nishihara et al., 2018).

Since ROS biological function is still controversial, several studies have implemented diverse strategies to modulate their production. A classic model to investigate the cytotoxic effect of $\mathrm{O}_{2}^{--}$and $\mathrm{H}_{2} \mathrm{O}_{2}$ is the use of xanthine oxidase (XOD) in combination with xanthine; XOD acts aerobically upon xanthine generating $\mathrm{O}_{2}^{--}$and $\mathrm{H}_{2} \mathrm{O}_{2}$ (Alvarez et al., 2015; Blondin et al., 1997; Goud et al., 2008; Lamirande and Gagnon, 1993). Then, the inclusion of catalase to the xanthine + XOD system would mainly generate $\mathrm{O}_{2} \cdot-$ due to the transformation of $\mathrm{H}_{2} \mathrm{O}_{2}$ into $\mathrm{H}_{2} \mathrm{O}$ and $\mathrm{O}_{2}$ mediated by catalase (Von Mengden et al., 2020). Another strategy to modulate endogenous ROS levels could be to regulate NAPDH oxidases, the only enzymes whose primary function seem to be ROS production, using specific inhibitors such as diphenyl iodonium (IDP) (Altenhöfer et al., 2015).

To date, most of the studies carried out during oocyte in vitro maturation (IVM) have been focused on the ef- fects of exogenous ROS. We hypothesize that IVM could be influenced by endogenous ROS levels, which in turn might be modified by the presence of enzymatic and nonenzymatic scavenger systems present in the follicular fluid. Therefore, the aim of this study was to examine the effect of varying endogenous ROS levels during IVM on the process of bovine oocyte maturation by adding different enzymatic antioxidant (catalase or SOD + catalase or IDP) or pro-oxidant systems (xanthine + XOD or xanthine + XOD + catalase) to the culture medium.

\section{Material and methods}

\section{Reagents and media}

The materials used in these experiments were obtained from Sigma-Aldrich (St. Louis, Missouri), unless otherwise indicated.

\section{Immature cumulus-oocyte complexes recovery and classification}

Bovine ovaries were collected in an abattoir from slaughtered cows and kept warm $\left(30-33^{\circ} \mathrm{C}\right)$ during the 2-h transport to the laboratory. Ovaries were washed in physiological saline containing 100,000 IU penicillin and $100 \mathrm{mg}$ streptomycin per liter. COCs were recovered by aspiration of antral follicles (2-5 mm) and selected using a stereomicroscope. Only good quality oocytes, which presented a homogenous cytoplasm, integer zona pellucida and completely surrounded by a compact and thick cumulus (class A, Cetica et al., 1999) were used.

\section{Oocyte in vitro maturation using different pro- oxidant or antioxidant enzymatic systems \\ COCs were matured in TCM 199 supplemented with} $5 \%(\mathrm{v} / \mathrm{v})$ fetal calf serum (FCS), $50 \mu \mathrm{g} / \mathrm{ml}$ gentamicin sulfate, $0.2 \mu \mathrm{g} / \mathrm{ml} \mathrm{FSH}$ and $2 \mu \mathrm{g} / \mathrm{ml} \mathrm{LH}$ during $22 \mathrm{~h}$ (control medium) with or without the addition of different modulators of ROS production.

To analyze the effect of $\mathrm{H}_{2} \mathrm{O}_{2}$ or $\mathrm{O}_{2}^{--}$, the control medium was supplemented with: (i) $2 \mathrm{mM}$ xanthine $+1 \mathrm{IU} /$ $\mathrm{ml} \mathrm{XOD}\left(\mathrm{H}_{2} \mathrm{O}_{2}\right.$ generating system); (ii) $50 \mathrm{IU} / \mathrm{ml}$ catalase $\left(\mathrm{H}_{2} \mathrm{O}_{2}\right.$ scavenger); (iii) $2 \mathrm{mM}$ xanthine $+1 \mathrm{IU} / \mathrm{ml} \mathrm{XOD}$ $+50 \mathrm{IU} / \mathrm{ml}$ catalase $\left(\mathrm{O}_{2} \cdot-\right.$ generating system); (iv) $50 \mathrm{IU} /$ $\mathrm{ml}$ catalase $+100 \mathrm{UI} / \mathrm{ml}$ SOD $\left(\mathrm{O}_{2}^{--}\right.$scavenger) (Alvarez et al., 2015). To analyze the participation of NADPH oxidase, the control medium was supplemented with $2 \mu \mathrm{M}$ IDP (NADPH oxidase inhibitor, O'Flaherty et al., 2005). The concentrations used for the different treatments were selected as they proved to have an effect in the above cited works published by our group in porcine COCs and bovine sperm, respectively.

\section{Determination of ROS production}

To measure ROS production COC samples were collected from the different maturation media at $0,6,12,18$ and $22 \mathrm{~h}$. Oocytes were completely denuded by gentle pipetting in phosphate buffer saline supplemented with 
$0.3 \mathrm{mg} / \mathrm{ml}$ polyvinyl alcohol (PBS-PVA). Then, denuded oocytes were incubated in PBS-PVA in the presence of $5 \mu \mathrm{M}$ 2',7'-dichlorodihydrofluorescein diacetate (DCHFDA) for 30 min (Morado et al., 2009). Additionally, other groups of COCs were collected at the same time points, they were denuded and incubated in PBS-PVA in the presence of $0.12 \mu \mathrm{M}$ fluorescein diacetate (FDA) for $15 \mathrm{~min}$, to measure esterase activity.

After their exposure to the respective fluorochrome, both COC samples were washed in PBS-PVA and mounted on glass slides. Fluorescence was measured by means of digital microphotographs in a Jenamed II epifluorescence microscope using 450-490 nM (excitation) and $520 \mathrm{nM}$ (emission) filters. Photographs were analyzed using the software Image J 1.240. As fluorescence levels detected by DCHFDA are dependent on esterase activity, a ratio between the brightness of each oocyte measured by DCHFDA and the mean brightness detected by FDA at each time point was considered a more accurate indicator of the relative ROS level for each oocyte (Morado et al., 2009). ROS levels were expressed as arbitrary ROS units/oocyte/minute.

\section{Oocyte denudation and nuclear status evaluation}

After $22 \mathrm{~h}$ maturation, COC samples were collected, denuded as previously described and incubated in a $10 \mathrm{mg} /$ $\mathrm{ml}$ sodium citrate hypotonic solution for $15 \mathrm{~min}$, fixed on a glass slide with acetic acid-ethanol solution, stained with $5 \%$ Giemsa for $15 \mathrm{~min}$ and observed at x400 and x1000 magnification under a light microscope to determine their nuclear status. Meiotic maturation rate was determined by the number of oocytes which presented metaphase II chromosome configuration at $22 \mathrm{~h}$ (Morado et al., 2009).

\section{In vitro fertilization of matured oocytes}

Matured oocyte activation was carried out using frozen-thawed Holstein bull semen from a male of proven fertility. The semen straws were thawed at $37^{\circ} \mathrm{C}$ in modified synthetic oviductal fluid (mSOF) (Takahashi and First, 1992) supplemented with $10 \mathrm{mmol} / 1$ theophylline, centrifuged at $500 \mathrm{~g}$ twice for 5 minutes and resuspended in $\mathrm{mSOF}$ to a final concentration of $2 \times 10^{6}$ spermatozoa/ $\mathrm{ml}$ (Morado et al., 2013). Co-incubation of COCs and spermatozoa was performed in mSOF supplemented with $10 \mathrm{UI} / \mathrm{ml}$ heparin and $5 \mathrm{mg} / \mathrm{ml}$ bovine serum albumin (BSA) under mineral oil at $39^{\circ} \mathrm{C}$ in a humidified atmosphere with $5 \% \mathrm{CO}_{2}$ during $24 \mathrm{~h}$. Then, presumptive zygotes were transferred to mSOF supplemented with 30 $\mu \mathrm{l} / \mathrm{ml}$ essential amino acids, $1 \mu \mathrm{l} / \mathrm{ml}$ nonessential amino acids, $2 \mu \mathrm{mol} / \mathrm{ml} \mathrm{L-glutamine,} 5 \mathrm{mg} / \mathrm{ml} \mathrm{BSA}$, $5 \%(\mathrm{v} / \mathrm{v})$ FBS and $50 \mu \mathrm{g} / \mathrm{ml}$ gentamicin sulfate and the cleavage rate was evaluated by the number of embryos which presented two or more blastomeres after $24 \mathrm{~h}$.

\section{Statistical analysis}

ROS levels were expressed as mean \pm SEM and compared by a $5 \times 2$ factorial design in the experiments using NADPH oxidase inhibitor and by a $5 \times 3$ factorial design in the experiments using $\mathrm{H}_{2} \mathrm{O}_{2}$ and $\mathrm{O}_{2}{ }^{-}$generating and scavenging systems. Meiotic maturation and cleavage rates between treatments were compared using chi-square analysis for non-parametric data. The $\mathrm{P}$ value used to determine significance in all tests was 0.05 .

\section{Results}

ROS production in the presence of different prooxidant or antioxidant enzymatic systems

Time dependent fluctuations were detected in ROS levels throughout IVM, presenting similar patterns in control oocytes and in those cultured with the different treatments. Neither the $\mathrm{H}_{2} \mathrm{O}_{2}$ generating system nor its scavenger caused significant changes in ROS production in bovine oocytes with respect to the control (xanthine + XOD or catalase, respectively, Figure 1). The same result was observed in the presence of the NADPH oxidase inhibitor (IDP, Figure 3) and the $\mathrm{O}_{2}{ }^{--}$scavenger (SOD + catalase, Figure 2). However, for oocytes cultured in the presence of the $\mathrm{O}_{2}^{-}$generating system (xanthine + XOD + catalase) a significant increase in ROS levels was detected at both 6 and $18 \mathrm{~h}$ compared with the control (Figure 2, $\mathrm{P}<0.05$ ).

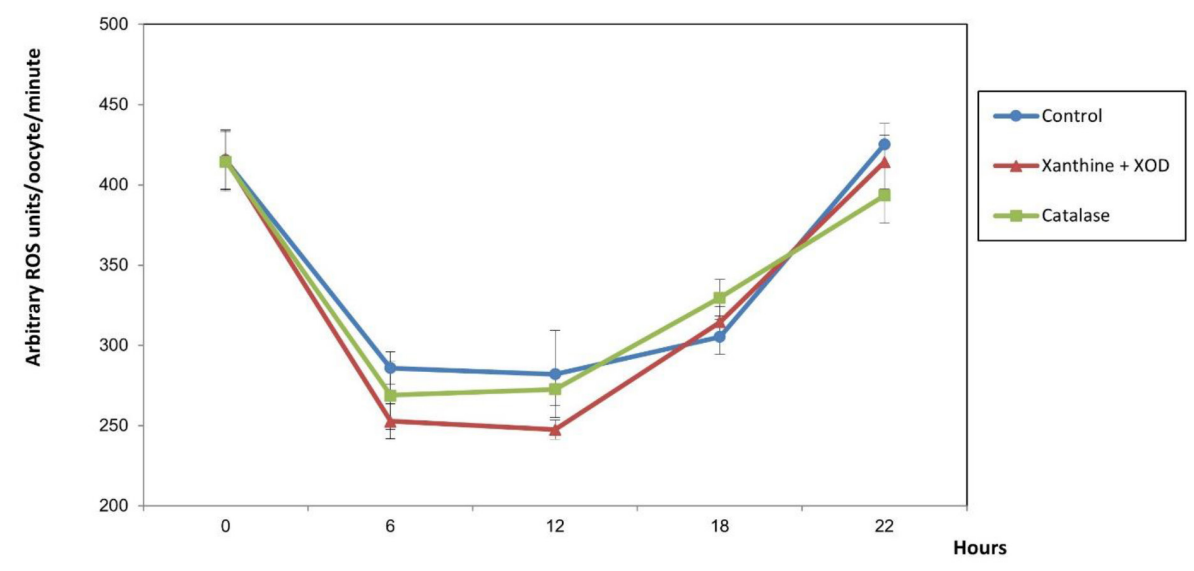

Figure 1. ROS production of bovine oocytes during in vitro maturation with or without the addition of xanthine + xanthine oxidase (XOD) or catalase (generators or scavenger of $\mathrm{H}_{2} \mathrm{O}_{2}$, respectively). Values are expressed as mean arbitrary units/oocyte/minute $\pm \mathrm{SEM}$. $\mathrm{n}=217$ oocytes, in 3 replicates. No significant differences were detected between treatments 
Esterase activity was evaluated by the FDA assay at different time points in oocytes cultured in control medium and in the presence of $\mathrm{H}_{2} \mathrm{O}_{2}$ and $\mathrm{O}_{2}^{--}$generating and scavenging systems. No time dependent differences were detected in esterase activity in control oocytes nor in those cultured with the different treatments. Moreover, no significant differences were observed between treatments (data not shown), so the fluctuations observed in Figures 1 to 3 can be exclusively attributed to the variations in endogenous ROS levels detected during IVM.
Maturation rates in the presence of different prooxidant or antioxidant enzymatic systems

As shown in Table 1, oocytes cultured in the presence of both generators and scavengers of $\mathrm{H}_{2} \mathrm{O}_{2}$ and $\mathrm{O}_{2}-$ - presented a significantly lower meiotic maturation rate compared with those matured in the control medium $(\mathrm{P}<0.05)$. On the other hand, meiotic maturation rates between oocytes matured in the presence of IDP and the control showed no significant differences.

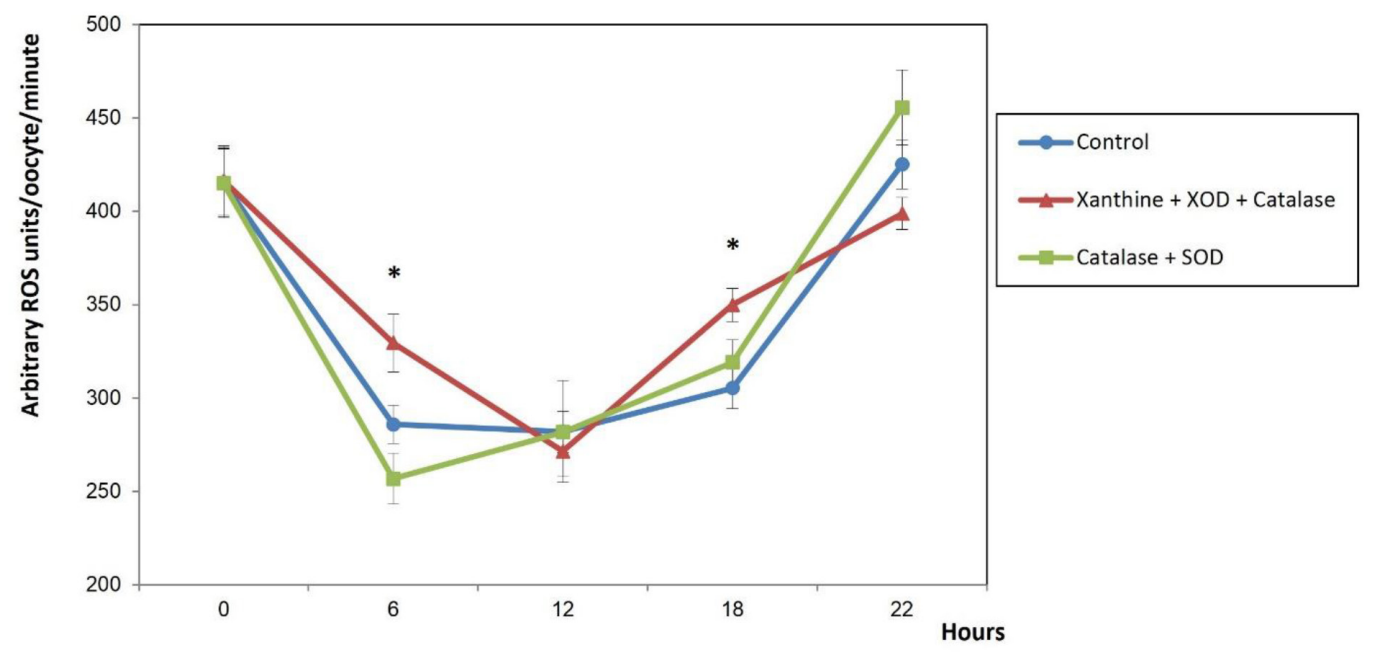

Figure 2. ROS production of bovine oocytes during in vitro maturation with or without the addition of xanthine + xanthine oxidase (XOD) + catalase or catalase + superoxide dismutase (SOD) (generators or scavengers of $\mathrm{O}_{2}^{--}$, respectively). Values are expressed as mean arbitrary units/ oocyte/minute \pm SEM. $\mathrm{n}=225$ oocytes, in 3 replicates. *Significant difference between control and treatment $(\mathrm{P}<0.05)$

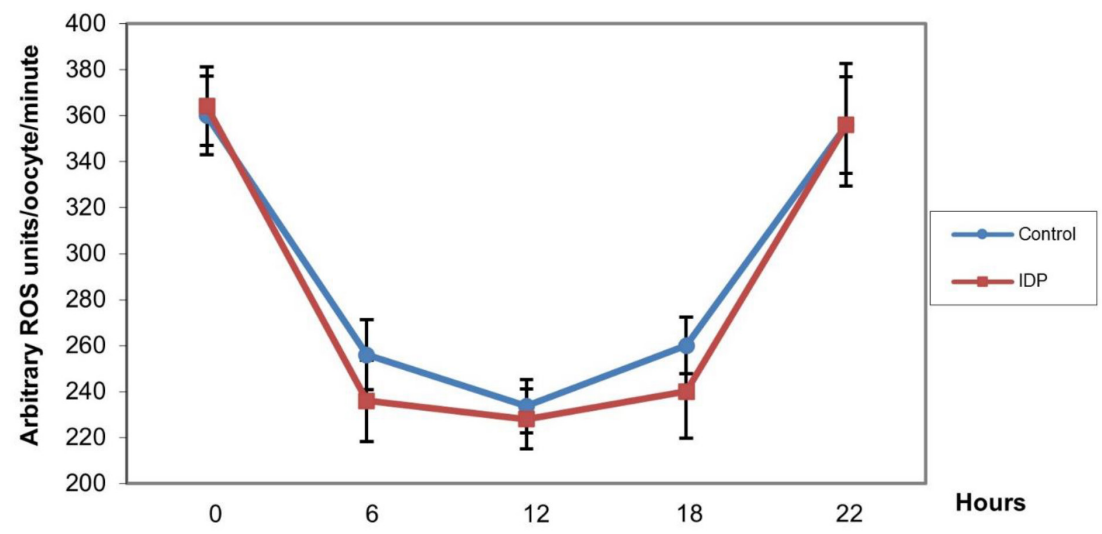

Figure 3. ROS production of bovine oocytes during in vitro maturation with or without the addition of diphenyl iodonium (inhibitor of NADPH oxidase). Values are expressed as mean arbitrary units/oocyte/minute \pm SEM. $n=250$ oocytes, in 3 replicates. No significant differences were detected between treatments

Table 1. Meiotic maturation rates for bovine oocytes matured in the presence of different pro-oxidant or scavenger enzymatic systems

\begin{tabular}{|c|c|c|c|c|}
\hline Treatment & $\begin{array}{c}\text { Germinal vesicle } \\
(\%)\end{array}$ & $\begin{array}{c}\text { GVB - Metaphase I } \\
(\%)\end{array}$ & $\begin{array}{c}\text { Metaphase II } \\
(\%)\end{array}$ & $\mathrm{N}$ \\
\hline Control & $3(7.5)$ & - & $37(92.5) \mathrm{a}$ & 40 \\
\hline Xanthine + Xanthine oxidase & $5(12.5)$ & $16(40)$ & $19(47.5) b$ & 40 \\
\hline Catalase & $4(10)$ & $15(37.5)$ & $21(52.5) b$ & 40 \\
\hline Xanthine + Xanthine oxidase + Catalase & $4(10)$ & $10(25)$ & $26(65) b$ & 40 \\
\hline Catalase + Superoxide dismutase & $4(10)$ & $14(35)$ & $22(55) \mathrm{b}$ & 40 \\
\hline Diphenyl iodonium & $6(15)$ & - & $34(85) \mathrm{a}$ & 40 \\
\hline
\end{tabular}

$\mathrm{a}, \mathrm{b}$ - significant difference between treatments $(\mathrm{P}<0.05) . \mathrm{n}=240$ oocytes in 3 replicates. GVB: germinal vesicle breakdown. 
Cleavage rate in bovine oocytes in vitro matured with a NADPH oxidase inhibitor

As the systems used to generate and scavenge $\mathrm{H}_{2} \mathrm{O}_{2}$ and $\mathrm{O}_{2}{ }_{2}^{--}$significantly reduced the meiotic maturation rate of bovine oocytes, cleavage rate was only evaluated in those oocytes matured in the presence of IDP. This NADPH oxidase inhibitor significantly decreased cleavage rate ( 8 cleaved embryos/45 oocytes; $18 \%$ ) in comparison with the control group (36 cleaved embryos/45 oocytes; $80 \%)(\mathrm{P}<0.05 ; 3$ replicates $)$.

\section{Discussion}

As far as we know, this is the first study which examined the effect of varying endogenous ROS levels during bovine oocyte IVM by adding $\mathrm{H}_{2} \mathrm{O}_{2}$ and $\mathrm{O}_{2}-$ enzymatic generating and scavenging systems to the culture medium. Our results suggest that ROS may exert an essential function during IVM, since the addition of both pro-oxidant and antioxidant systems significantly reduced meiotic maturation or cleavage rates in bovine oocytes.

Several studies have reported that cumulus cells have an active participation in the regulation of ROS levels within the COC. For instance, catalase is scarce in oocytes but is synthesized in cumulus cells and protects the oocyte by metabolizing $\mathrm{H}_{2} \mathrm{O}_{2}$ into non-reactive molecules (Cetica et al., 2001; Von Mengden et al., 2020). Therefore, the increase in $\mathrm{H}_{2} \mathrm{O}_{2}$ generated by the addition of xanthine + XOD could have been compensated by the high catalase activity in cumulus cells, thus explaining why we did not observe an increase in ROS production in the oocyte with this treatment. We may also hypothesize that this is the reason why the addition of this enzyme to the maturation medium did not further reduce ROS levels. Ali et al. (2003) also evaluated the use of catalase during bovine oocyte IVM finding that the supplementation of maturation media with extracellular antioxidants did not exert any beneficial effect, probably because the cumulus cells isolate the oocyte from the extracellular medium. Alvarez et al. (2015) also reported that the addition of this extracellular scavenger did not decrease ROS levels in matured porcine oocytes nor improve nuclear or cytoplasmic maturation rates.

In contrast to the modulation of $\mathrm{H}_{2} \mathrm{O}_{2}$ levels, the increase in $\mathrm{O}_{2}^{--}$production using xanthine $+\mathrm{XOD}+$ catalase coincided with a significant increase in endogenous ROS levels during IVM. This could be explained by the differences in the expression of SOD isoforms between oocytes and cumulus cells. This enzyme presents three isoforms, the cytosolic $\mathrm{Cu}, \mathrm{Zn}-\mathrm{SOD}$ (SOD1), the mitochondrial Mn-SOD (SOD2) and the extracellular SOD3 (Von Mengden et al., 2020). A study performed in bovine antral follicles reported that oocytes and cumulus cells express SOD2 and SOD3, while SOD1 was only expressed in oocytes (Combelles et al., 2010). Therefore, cumulus cells would not be capable of scavenging the increase in $\mathrm{O}_{2}^{--}$, which would then enter the oocyte through different membrane transporters explaining the increase observed in ROS levels. In coincidence, it was observed that SOD exerts a lower specific activity in cumulus cells with respect to oocytes in bovines (Cetica et al., 2001). These results could also indicate that the xanthine + XOD + catalase enzymatic system is more important than the xanthine + XOD system in the generation of oxidative stress.

$\mathrm{O}_{2}^{-}$and $\mathrm{H}_{2} \mathrm{O}_{2}$ produced in the culture media may be transported inside cells by aquaporins and chloride channel-3, respectively (Bienert et al., 2006; van der Vliet, 2008). Once inside the oocyte, both molecules may activate different signaling pathways, including those which involve the action of mitogen-activated protein kinases (MAPK) (Hancock et al., 2001). MAPK superfamily members have critical roles in the meiotic process acting as signaling molecules and affecting spindle assembly and microtubule organization in oocytes, also participating in cumulus cell expansion, cytoplasmic maturation and embryo development (Song et al., 2018). The potential increase in the entry of $\mathrm{H}_{2} \mathrm{O}_{2}$ and $\mathrm{O}_{2}{ }^{-}$to the oocyte produced by the added pro-oxidant enzymatic systems could stimulate an even greater generation of $\mathrm{H}_{2} \mathrm{O}_{2}$ in the mitochondria, possibly also through the MAPK pathway, initiating a cycle of oxidative stress amplification that could lead to cell death (Egea et al., 2017). This would explain that while the removal of $\mathrm{H}_{2} \mathrm{O}_{2}$ and $\mathrm{O}_{2}^{--}$could affect the meiotic progress of the oocytes by interfering with intracellular signaling systems, their excess would also affect the process by generating oxidative damage in the oocyte.

Besides the antioxidant enzymes mentioned above, bovine oocytes also express six different peroxiredoxins (PRDX1 to PRDX6), which are involved in antioxidant protection and cell signaling. These enzymes are expressed during oocyte maturation, in both the oocyte and the cumulus cells, as well as in early embryonic development (Leyens et al., 2004 a). Particularly, the expression of PRDX6 in the oocyte and cumulus cells depends on the interaction between these two types of cells, indicating a paracrine effect and cell communication via gap junctions (Leyens et al., 2004 b), while the expression of PRDX1 exhibits a positive correlation with the developmental competence of bovine oocytes derived from follicles of different sizes (Mourot et al., 2006). During IVM, this PRDX does not only participate in the prevention of oxidative damage, but also participates in signal transduction systems involved in cumulus expansion (Leyens et al., 2004 b).

Although NOX is a physiological source of $\mathrm{O}_{2}^{--}$and $\mathrm{H}_{2} \mathrm{O}_{2}$, its inhibition by the use of IDP did not decrease ROS levels as expected, suggesting that this enzyme may not have a major participation in the generation of oxidative stress during IVM. The participation of NOX enzymes in the ovulation process has been studied in Drosophila, where it has been determined that NOX regulates follicle rupture by generating $\mathrm{O}_{2}^{--}$which is later converted to $\mathrm{H}_{2} \mathrm{O}_{2}$ by SOD3. In this way, $\mathrm{H}_{2} \mathrm{O}_{2}$ acts as 
a key molecule in the regulation and control of ovulation, and, as NOX proteins are also expressed in mammalian follicles, this NOX dependent pathway could play a conserved role in regulating ovulation in other species ( $\mathrm{Li}$ et al., 2018). In porcine oocytes the use of IDP during IVM significantly reduced the maturation rate, as well as embryo development, probably due to a lower resumption of meiosis caused by a lower activity of the pentosephosphate pathway (Herrick et al., 2006).

Despite the observation that most of the evaluated enzymatic systems did not alter endogenous ROS levels, the maturation rates were significantly lower in comparison with the control group, except for the oocytes treated with IDP. The use of IDP during IVM did cause a significantly lower cleavage rate compared with the control, indicating a reduction in the cytoplasmic maturation of these oocytes, which could be related with an excessive decrease in ROS, a not so familiar situation known as reductive stress (Altenhöfer et al., 2015). Therefore, our results suggest that physiological ROS levels would be necessary for both nuclear and cytoplasmatic bovine oocyte maturation and its regulation would involve the participation of the studied enzymatic systems.

In conclusion, the modulation of endogenous oocyte ROS levels by the use of different pro-oxidant or antioxidant enzymatic systems in the culture media proved to be a useful tool to increase the understanding of the physiological role of ROS in bovine oocyte cytoplasmic and nuclear maturation, the xanthine $+\mathrm{XOD}+$ catalase system being more relevant than xanthine + XOD in the generation of oxidative stress. These enzymatic systems allowed us to demonstrate that even when an increase in the presence of $\mathrm{O}_{2}^{-}$and $\mathrm{H}_{2} \mathrm{O}_{2}$ is detrimental for oocyte quality, their excessive removal may also alter nuclear maturation, decreasing oocyte competence. Our results also represent the first study which determined the essential role of NADPH oxidase during bovine oocyte IVM; although its inhibition did not alter oocyte quality in terms of ROS levels, it did reduce oocyte cleavage rates.

Our findings indicate that ROS should not only be considered as harmful molecules responsible for oxidative stress, but also as physiological compounds responsible for activating signaling pathways necessary for an adequate maturation and activation in bovine oocytes. Further studies are necessary to determine the precise way in which these reactive species participate in signaling pathways such as those related with MAPK, the maturation promoting factor and other kinase dependent cyclins, which could be involved in both processes in the bovine.

\section{Acknowledgements}

Authors would like to thank Cocarsa Slaughterhouse for the ovaries provided.

\section{Conflict of interest statement}

The authors declare that there is no conflict of interest that could prejudice the impartiality of the research reported.

\section{References}

Aitken R.J. (2020). Impact of oxidative stress on male and female germ cells: implications for fertility. Reproduction, 159: R189-R201.

Ali A.A., Bilodeau J.F., Sirard M.A. (2003). Antioxidant requirements for bovine oocytes varies during in vitro maturation, fertilization and development. Theriogenology, 59: 939-949.

Ali I., Liu H.X., Zhong-Shu L., Dong-Xue M., Xu L., Shah S.Z.A., Ullah O., Nan-Zhu F. (2018). Reduced glutathione alleviates tunicamycin-induced endoplasmic reticulum stress in mouse preimplantation embryos. J. Reprod. Dev., 64: 15-24.

Altenhöfer S., Radermacher K.A., Kleikers P.W.M., Wingler K., Schmidt H.H.H.W. (2015). Evolution of NADPH oxidase inhibitors: Selectivity and mechanisms for target engagement. Antioxid. Redox Sign., 23: 406-427.

Alvarez G., Morado S., Soto M., Dalvit G., Cetica P. (2015). The control of reactive oxygen species influences porcine oocyte in vitro maturation. Reprod. Domest. Anim., 50: 200-205.

Bienert G.P., Schjoerring J.K., Jahn T.P. (2006). Membrane transport of hydrogen peroxide. Biochim. Biophys. Acta - Biomembr., 1758: 994-1003.

Blondin P., Coenen K., Sirard M.A. (1997). The impact of reactive oxygen species on bovine sperm fertilizing ability and oocyte maturation. J. Androl., 18: 454-460.

Buck T., Hack C.T., Berg D., Berg U., Kunz L., Mayerhofer A. (2019). The NADPH oxidase 4 is a major source of hydrogen peroxide in human granulosa-lutein and granulosa tumor cells. Sci. Rep., 9: 1-11.

Carbone M.C., Tatone C., Delle Monache S., Marci R., Caserta D., Colonna R., Amicarelli F. (2003). Antioxidant enzymatic defences in human follicular fluid: Characterization and age-dependent changes. Mol. Hum. Reprod., 9: 639-643.

Cetica P.D., Dalvit G.C., Beconi M.T. (1999). Study of evaluation criteria used for in vitro bovine oocyte selection and maturation. Biocell, 23: 125-133.

Cetica P.D., Pintos L.N., Dalvit G.C., Beconi M.T. (2001). Antioxidant enzyme activity and oxidative stress in bovine oocyte in vitro maturation. IUBMB Life, 51: 57-64.

Christou-Kent M., Dhellemmes M., Lambert E., Ray P.F., Arnoult C. (2020). Diversity of RNA-binding proteins modulating post-transcriptional regulation of protein expression in the maturing mammalian oocyte. Cells, 9: 662.

Combelles C.M.H., Holick E.A., Paolella L.J., Walker D.C., Wu Q. (2010). Profiling of superoxide dismutase isoenzymes in compartments of the developing bovine antral follicles. Reproduction, 139: 871-881.

Cui M.S., Wang X.L., Tang D.W., Zhang J., Liu Y., Zeng S.M. (2011). Acetylation of H4K12 in porcine oocytes during in vitro aging: Potential role of ooplasmic reactive oxygen species. Theriogenology, 75: 638-646.

Egea J., Fabregat I., Frapart Y.M., Ghezzi P., Görlach A., Kietzmann T., Kubaichuk K., Knaus U.G., et al. (2017). European contribution to the study of ROS: A summary of the findings and prospects for the future from the COST action BM1203 (EU-ROS). Redox Biol., 13: 94-162.

El-Shahat K.H., Kandil M. (2012). Antioxidant capacity of follicular fluid in relation to follicular size and stage of estrous cycle in buffaloes. Theriogenology, 77: 1513-1518.

García-Martínez T., Vendrell-Flotats M., Martínez-Rodero I., Ordóñez-León E.A., Álvarez-Rodríguez M., López-Béjar M., Yeste M., Mogas T. (2020). Glutathione ethyl ester protects in vitro-maturing bovine oocytes against oxidative stress induced by subsequent vitrification/warming. Int. J. Mol. Sci., 21: 1-26.

Goud A.P., Goud P.T., Diamond M.P., Gonik B., Abu-Soud H.M. (2008). Reactive oxygen species and oocyte aging: Role of superoxide, hydrogen peroxide, and hypochlorous acid. Free Radic. Biol. Med., 44: 1295-1304.

Gupta S., Choi A., Yu H.Y., Czerniak S.M., Holick E.A., Paolella L.J., Agarwal A., Combelles C.M.H. (2011). Fluctuations in total antioxidant capacity, catalase activity, and hydrogen peroxide levels of follicular fluid during bovine folliculogenesis. Reprod. Fertil. Dev., 23: 673-680. 
Hancock J.T., Desikan R., Neill S.J. (2001). Role of reactive oxygen species in cell signalling pathways. Biochem. Soc. Trans., 29: 345-349.

Herrick J.R., Brad A.M., Krisher R.L. (2006). Chemical manipulation of glucose metabolism in porcine oocytes: Effects on nuclear and cytoplasmic maturation in vitro. Reproduction, 131: 289-298.

Kala M., Shaikh M.V., Nivsarkar M. (2017). Equilibrium between anti-oxidants and reactive oxygen species: a requisite for oocyte development and maturation. Reprod. Med. Biol., 16: 28-35.

Lamirande De E., Gagnon C. (1993). A positive role for the superoxide anion in triggering hyperactivation and capacitation of human spermatozoa. Int. J. Androl., 16: 21-25.

Leyens G., Knoops B., Donnay I. (2004 a). Expression of peroxiredoxins in bovine oocytes and embryos produced in vitro. Mol. Reprod. Dev., 69: 243-251.

Leyens G., Verhaeghe B., Landtmeters M., Marchandise J., Knoops B., Donnay I. (2004 b). Peroxiredoxin 6 is upregulated in bovine oocytes and cumulus cells during in vitro maturation: role of intercellular communication. Biol. Reprod., 71: 1646-1651.

Li W., Young J.F., Sun J. (2018). NADPH oxidase-generated reactive oxygen species in mature follicles are essential for Drosophila ovulation. Proc. Natl. Acad. Sci., 115: 7765-7770.

Lopes A., Lane M., Thompson J.G. (2010). Oxygen consumption and ROS production are increased at the time of fertilization and cell cleavage in bovine zygotes. Human Reprod., 25: 2762-2773.

Von Mengden L., Klamt F., Smitz J. (2020). Redox biology of human cumulus cells: basic concepts, impact on oocyte quality, and potential clinical use. Antioxid. Redox Sign., 32: 522-535.

Morado S.A., Cetica P.D., Beconi M.T., Dalvit G. C. (2009). Reactive oxygen species in bovine oocyte maturation in vitro. Reprod. Fert. Develop., 21: 608-614.

Morado S., Cetica P., Beconi M., Thompson J.G., Dalvit G. (2013). Reactive oxygen species production and redox state in parthenogenetic and sperm-mediated bovine oocyte activation. Reproduction, 145: 471-478.

Mouatassim El S., Guérin P., Ménézo Y. (1999). Expression of genes encoding antioxidant enzymes in human and mouse oocytes during the final stages of maturation. Mol. Hum. Reprod., 5: $720-725$.
Mourot M., Dufort I., Gravel C., Algriany O., Dieleman S., Sirard M.A. (2006). The influence of follicle size, FSH-enriched maturation medium, and early cleavage on bovine oocyte maternal mRNA levels. Mol. Reprod. Dev., 73: 1367-1379.

Nishihara T., Matsumoto K., Hosoi Y., Morimoto Y. (2018). Evaluation of antioxidant status and oxidative stress markers in follicular fluid for human in vitro fertilization outcome. Reprod. Med. Biol., 17: 481-486.

O’Flaherty C., Breininger E., Beorlegui N., Beconi M.T. (2005). Acrosome reaction in bovine spermatozoa: Role of reactive oxygen species and lactate dehydrogenase C4. Biochim. Biophys. Acta Gen. Subj., 1726: 96-101.

Pandey A.N., Chaube S. K. (2014). A moderate increase of hydrogen peroxide level is beneficial for spontaneous resumption of meiosis from diplotene arrest in rat oocytes cultured in vitro. Biores. Open Access, 3: 183-191.

Song B.S., Jeong P.S., Lee J.H., Lee M.H., Yang H.J., Choi S.A., Lee H.Y., Yoon S.B., Park Y.H., et al., (2018). The effects of kinase modulation on in vitro maturation according to different cumulus oocyte complex morphologies. PLoS One, 13: 1-20.

Takahashi Y., First N.L. (1992) In vitro development of bovine onecell embryos: influence of glucose, lactate, pyruvate, amino acids and vitamins. Theriogenology, 37: 963-978.

Vandaele L., Thys M., Bijttebier J., Van Langendonckt A., Donnay I., Maes D., Meyer E., Van Soom A. (2010). Short-term exposure to hydrogen peroxide during oocyte maturation improves bovine embryo development. Reproduction, 139: 505-511.

Velez-Pardo C., Tarazona Morales A., Jimenez Del Rio M., OliveraAngel M. (2007). Endogenously generated hydrogen peroxide induces apoptosis via mitochondrial damage independent of NF$\kappa \mathrm{B}$ and $\mathrm{p} 53$ activation in bovine embryos. Theriogenology, 67: 1285-1296.

van der Vliet A. (2008). NADPH oxidases in lung biology and pathology: Host defense enzymes, and more. Free Radic. Biol. Med., 44: 938-955.

Received: 1 VI 2021

Accepted: 29 IX 2021 